\title{
Restoring Size Consistency of Approximate Functionals Constructed from the Adiabatic Connection
}

\author{
Stefan Vuckovic, ${ }^{* \dagger}{ }^{\dagger}$ Paola Gori-Giorgi, ${ }^{\dagger}$ Pabio Della Sala, ${ }^{\ddagger}, \S_{(0)}$ and Eduardo Fabiano ${ }^{\ddagger}, \S_{(0)}$ \\ ${ }^{\dagger}$ Department of Theoretical Chemistry and Amsterdam Center for Multiscale Modeling, FEW, Vrije Universiteit, De Boelelaan 1083, \\ 1081HV Amsterdam, The Netherlands \\ ${ }^{\ddagger}$ Institute for Microelectronics and Microsystems (CNR-IMM), Via Monteroni, Campus Unisalento, 73100 Lecce, Italy \\ ${ }^{\S}$ Center for Biomolecular Nanotechnologies @UNILE, Istituto Italiano di Tecnologia, Via Barsanti, I-73010 Arnesano, Italy
}

Supporting Information

ABSTRACT: Approximate exchange-correlation functionals built by modeling in a nonlinear way the adiabatic connection $(\mathrm{AC})$ integrand of density functional theory have many attractive features, being virtually parameter-free and satisfying different exact properties, but they also have a fundamental flaw: they violate the size-consistency condition, crucial to evaluate interaction energies of molecular systems. We show that size consistency in the AC-based functionals can be restored in a very simple way at no extra computational cost. Results on a large set of benchmark molecular interaction energies show that functionals based on the interaction strength interpolation approximations are significantly more accurate than second-order perturbation theory.

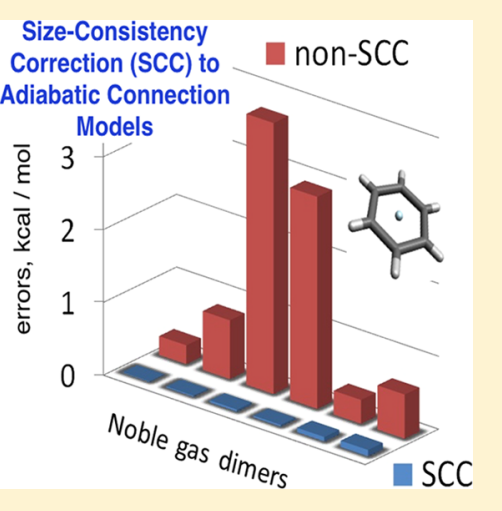

$\mathrm{W}$ ith applications that stretch from solid-state physics to biochemistry, Kohn-Sham density functional theory $(\mathrm{KS}-\mathrm{DFT})^{1}$ is presently the most employed electronic structure method. Although the theory is in principle exact, any practical implementation of KS-DFT must rely on approximations for the exchange-correlation (XC) functional, which should capture all of the many-body effects beyond the simple Hartree theory. Despite the existence of hundreds of different XC approximations $s^{2,3}$ and their widespread success in various disciplines, ${ }^{2}$ KS-DFT still encounters open issues, which hamper its overall predictive power ${ }^{2,4-6}$ and make the quest for better approximations a crucial research field for computational chemistry, solid-state physics, and materials science., ${ }^{2,4-6}$

The density-fixed adiabatic connection (AC) formalism ${ }^{7,8}$ provides an exact expression for the $\mathrm{XC}$ energy functional $E_{\mathrm{xc}}[\rho]$

$$
E_{\mathrm{xc}}[\rho]=\int_{0}^{1} W_{\lambda}[\rho] \mathrm{d} \lambda
$$

where $W_{\lambda}[\rho]$ is the AC integrand

$$
W_{\lambda}[\rho]=\left\langle\Psi_{\lambda}[\rho]\left|\hat{V}_{\mathrm{ee}}\right| \Psi_{\lambda}[\rho]\right\rangle-U[\rho]
$$

$\Psi_{\lambda}[\rho]$ is the Fermionic wave function with density $\rho(\mathbf{r})$ that minimizes the sum of the kinetic energy $\hat{T}$ and the electronelectron repulsion $\hat{V}_{e e}$ scaled by the coupling constant $\lambda$, and $U[\rho]$ is the Hartree energy. For small systems, $W_{\lambda}[\rho]$ has also been computed exactly through eq 2 . However, this requires the solution of the many-body Schrödinger equation..$^{9-11}$ Thus, for all practical purposes, $W_{\lambda}[\rho]$ must be approximated.
Equation 1 has been a fundamental milestone in guiding the construction of approximations. Early AC-based XC functionals used forms that depend linearly on some chosen input ingredients, such as the exchange energy from Hartree-Fock (HF) theory as the value to be recovered at $\lambda=0$, and semilocal approximations at some $\lambda=\lambda_{\mathrm{p}}$ between 0 and 1 . These forms are commonly used for the construction of hybrid ${ }^{12-14}$ and double-hybrid ${ }^{15-17}$ density functionals, resulting in mixing a fixed fraction of HF exchange and second-order perturbation theory with semilocal functionals. They often work well for main-group chemistry, but they show important limitations for various other problems as, for example, the chemistry of transition metals ${ }^{18}$ (where they even worsen the results with respect to simpler semilocal functionals), metal-molecule interfaces, ${ }^{19}$ and even noncovalent bonding (unless an ad hoc van der Waals correction is used). ${ }^{20,21}$ Their main disadvantage is that the mixing fractions are fixed and cannot adapt to different systems or to different parts of a system.

To address this problem, several models in which the input ingredients enter in a nonlinear way have been proposed. ${ }^{11,22-26}$ These latter forms do not need to rely on empiricism and can adapt automatically to the peculiarities of the system under study. Along these lines, Ernzerhof had proposed Padé forms for the $\lambda$ dependence of the AC integrand, ${ }^{22}$ which later were used for construction of the MCY

Received: April 5, 2018

Accepted: May 22, 2018

Published: May 22, 2018 
family of functionals that are constrained to be free of oneelectron self-interaction error. ${ }^{25,27}$ Another example of models that use input ingredients in a nonlinear way is provided by the interaction strength interpolation (ISI) functionals, which depend explicitly on the weak- and strong-coupling ingredients, ${ }^{23,24,28-30}$ essentially extending to nonuniform densities Wigner's $^{31,32}$ idea for approximating the energy of the homogeneous electron gas. Despite the advantages of the nonlinear forms over the linear ones, the former encounter a fundamental flaw: the XC functionals that are constructed from them are not size-consistent for systems composed of different species of fragments. ${ }^{27,33}$ This depends on the fact that these methods employ as input ingredients global quantities (integrated over all space). A route that is currently being explored addresses this issue by modeling the $\mathrm{AC}$ at each given spatial position $\mathbf{r}$, using energy densities $w_{\lambda}(\mathbf{r})^{26,34-36}$ instead of quantities integrated over all space. This strategy is very promising but does not allow using in a straightforward way semilocal ingredients ${ }^{26,33-39}$ because of the inherent ambiguity in the definition of energy densities, a problem shared with the construction of local hybrid functionals. $37,40,41$

In this Letter, we show that size consistency of the global (integrated over all space) AC forms in which the ingredients enter in a nonlinear way can be restored in a remarkably simple way, making it possible to obtain meaningful interaction energies at no additional computational cost.

Consider a system $M$ (e.g., a molecular complex) composed of a set of fragments $A_{i}$, with $i=1, \ldots, N$. The interaction energy is a key quantity in chemistry, and it is defined as

$$
E^{\mathrm{int}}(\mathrm{M})=E(\mathrm{M})-\sum_{i=1}^{N} E\left(\mathrm{~A}_{i}\right)
$$

where $E(M)$ is the energy of the bound system $M$ and $E\left(\mathrm{~A}_{i}\right)$ are the energies of the individual fragments. If we now compute the energy of a system $M^{*}$ made of the same fragments $A_{i}$ placed at a very large (infinite) distance from each other, any sizeconsistent method should give $E^{\text {int }}\left(M^{*}\right)=0$, or equivalently

$$
E\left(\mathrm{M}^{*}\right)=\sum_{i=1}^{N} E\left(\mathrm{~A}_{i}\right)
$$

We should stress at this point that size consistency in DFT is in general a very subtle issue, particularly when dealing with fragments with a degenerate ground state (e.g., open-shell atoms) as the (spin) density is no longer an intensive quantity. ${ }^{42,43}$ To disentangle this more general DFT problem from the one of size consistency of the nonlinear AC models, here we focus on the cases where the fragments have a nondegenerate ground state, ${ }^{44}$ considering noncovalent interactions.

The idea behind AC-based functionals is to use a certain number of input ingredients $W_{i}[\rho]$, constructing a $\lambda$-dependent function that interpolates between them. For example, many standard hybrid functionals model $W_{\lambda}[\rho]$ with functions of the kind

$$
W_{\lambda}^{\mathrm{hyb}}[\rho]=W_{\lambda}^{\mathrm{DFA}}[\rho]+\left(E_{\mathrm{x}}^{\mathrm{HF}}[\rho]-E_{\mathrm{x}}^{\mathrm{DFA}}[\rho]\right) \lambda^{n-1}
$$

where $W_{\lambda}^{\mathrm{DFA}}[\rho]$ is a given density functional approximation (usually a semilocal functional), with its exchange component $W_{\lambda=0}^{\mathrm{DFA}}[\rho]=E_{\mathrm{x}}^{\mathrm{DFA}}[\rho]$, and $E_{\mathrm{x}}^{\mathrm{HF}}[\rho]$ is the HF exchange energy.

These kinds of expressions, when inserted into eq 1 , yield a fixed fraction $1 / n$ of HF exchange energy mixed with a semilocal density functional approximation. Because the input ingredients, in this case, $W_{\lambda}^{\mathrm{DFA}}[\rho], E_{\mathrm{x}}^{\mathrm{HF}}[\rho]$, and $E_{\mathrm{x}}^{\mathrm{DFA}}[\rho]$, enter linearly in the model of eq 5 , the resulting XC functional automatically satisfies the size-consistency condition of eq 4 if the individual ingredients do.

As examples of approximations in which the ingredients enter in a nonlinear way, consider first the Padé $([1 / 1])$ form introduced by Ernzerhof ${ }^{22}$

$$
W_{\lambda}^{\mathrm{Pad}}[\rho]=a[\rho]+\frac{b[\rho] \lambda}{1+c[\rho] \lambda}
$$

with $a[\rho]=W_{0}[\rho]=E_{\mathrm{x}}^{\mathrm{HF}}[\rho], b[\rho]=W_{0}^{\prime}[\rho]$ (which can be obtained from second-order perturbation theory), and $c[\rho]=$ $\lambda_{\mathrm{p}}^{-1}-W_{0}^{\prime}[\rho]\left(W_{0}[\rho]-W_{\lambda_{\mathrm{p}}}^{\mathrm{DFA}}[\rho]\right)^{-1}$, where $W_{\lambda_{\mathrm{p}}}^{\mathrm{DFA}}[\rho]$ could be a semilocal functional at a chosen value $\lambda_{\mathrm{p}}$. We see immediately that in this case, even if the input quantities $W_{0}, W_{0}^{\prime}$, and $W_{\lambda_{\mathrm{p}}}$ satisfy the size-consistency condition of eq 4 , the resulting $\mathrm{XC}$ functional from eq 1 does not, because it is given by a nonlinear function $f^{\mathrm{Pad}}$ of these ingredients, $E_{\mathrm{xc}}^{\mathrm{Pad}}=f^{\mathrm{Pad}}\left(W_{0}, W_{0}^{\prime}, W_{\lambda_{\mathrm{p}}}\right)$.

Another example is the idea of Seidl and co-workers ${ }^{23,24}$ to build approximate $W_{\lambda}[\rho]$ by interpolating between its weak $(\lambda \rightarrow 0)$ and strong $(\lambda \rightarrow \infty)$ coupling expansions

$$
\begin{aligned}
& W_{\lambda \rightarrow 0}[\rho]=W_{0}[\rho]+\lambda W_{0}^{\prime}[\rho]+\ldots \\
& W_{\lambda \rightarrow \infty}[\rho]=W_{\infty}[\rho]+\frac{W_{\infty}^{\prime}[\rho]}{\sqrt{\lambda}}+\ldots
\end{aligned}
$$

which allows one to avoid bias toward the weakly correlated regime and to include more pieces of exact information. The $\lambda \rightarrow 0$ limit of eq 7 is provided by the exact exchange and the second-order perturbation theory, while the functionals $W_{\infty}[\rho]$ and $W_{\infty}^{\prime}[\rho]$ describe a floating Wigner crystal with a metric dictated by the density. ${ }^{28,45}$

Different formulas that interpolate between the limits of eqs 7 and 8 are available in the literature. ${ }^{23,24,26,28,46}$ As in the Padé example of eq 6, when these interpolation formulas are inserted into eq 1 they give an XC energy that is a nonlinear function of the four ingredients (or a subset thereof), $W_{0}[\rho], W_{0}^{\prime}[\rho]$, $W_{\infty}^{\prime}[\rho]$, and $W_{\infty}[\rho]$, appearing in eqs 7 and 8 .

It is clear from these examples that we can write a general $\mathrm{XC}$ functional obtained by modeling the $\mathrm{AC}$ as

$$
E_{\mathrm{xc}}^{\mathrm{ACM}}[\rho]=f^{\mathrm{ACM}}(\mathbf{W}[\rho])
$$

where $f^{\mathrm{ACM}}$ is a nonlinear function that results from the integration (via eq 1) of the given adiabatic connection model $(\mathrm{ACM})$ and $\mathbf{W}[\rho]=\left\{W_{1}[\rho], \ldots, W_{k}[\rho]\right\}$ is a compact notation for the $k$ input ingredients that have been used. Then we have

$$
\sum_{i=1}^{N} E_{\mathrm{xc}}^{\mathrm{ACM}}\left(\mathrm{A}_{i}\right)=\sum_{i=1}^{N} f^{\mathrm{ACM}}\left(\mathbf{W}\left(\mathrm{A}_{i}\right)\right)
$$

and

$$
E_{\mathrm{xc}}^{\mathrm{ACM}}\left(\mathrm{M}^{*}\right)=f^{\mathrm{ACM}}\left(\sum_{i=1}^{N} \mathbf{W}\left(\mathrm{A}_{i}\right)\right)
$$

This equation is one of the main points in this work. Although conceptually simple, it shows that the energy of a set of infinitely distant fragments $\left(\mathrm{M}^{*}\right)$ can be expressed as a function of the quantities of the isolated fragments. Notice that this holds in this special case because $f^{\mathrm{ACM}}$ is a function of global size-consistent ${ }^{44}$ quantities. For size-inconsistent wave function 
methods, such as CISD, this is usually not true, and the energy of $\mathrm{M}^{*}$ needs to be computed by performing an extra calculation with the fragments at a large distance, which might be tricky to do in practice.

Essentially, all of the models that have been proposed in the literature ${ }^{27}$ satisfy the condition

$$
f^{\mathrm{ACM}}(N \mathbf{W}(\mathrm{A}))=N f^{\mathrm{ACM}}(\mathbf{W}(\mathrm{A}))
$$

meaning that they are size-consistent when a system dissociates into equal fragments (size-extensivity). This is also a key difference with the size-consistency problem of wave function methods, which also arises in the case of equal fragments. However, when the $A_{i}$ are of different species, eqs 10 and 11 give in general different results, and attempts to make them equal for a nonlinear model have failed so far. ${ }^{27}$

As said, evaluating eq 10 or 11 has exactly the same computational cost as both equations only need the input ingredients for the individual fragments. The idea behind the size-consistency correction (SCC) is thus extremely simple, and it is related to discussions reported in refs 47-49; it consists of using the difference between eqs 11 and 10 to cancel the sizeconsistency error that is made when evaluating interaction energies from eq 3

$$
\Delta_{\mathrm{SCC}}=\sum_{i=1}^{N} f^{\mathrm{ACM}}\left(\mathbf{W}\left(\mathrm{A}_{i}\right)\right)-f^{\mathrm{ACM}}\left(\sum_{i=1}^{N} \mathbf{W}\left(\mathrm{A}_{i}\right)\right)
$$

Note that this correction is fundamentally different from a direct calculation of $\sum_{i} E\left(\mathrm{~A}_{i}\right)-E\left(\mathrm{M}^{*}\right)$ because, due to the use of eq 11 , only knowledge of the isolated fragments is required here, while there is no need to deal with the (possibly tricky) calculation of the supramolecular energy $M^{*}$.

Adding $\Delta_{\text {SCC }}$ to an interaction energy computed via eq 3 is equivalent to always evaluating interaction energies with respect to eq 11 instead of eq 10 , i.e.

$$
E_{\mathrm{xc}, \text { int }}^{\mathrm{ACM}, \mathrm{SCC}}[\rho]=f^{\mathrm{ACM}}(\mathbf{W}(\mathrm{M}))-f^{\mathrm{ACM}}\left(\sum_{i=1}^{N} \mathbf{W}\left(\mathrm{A}_{i}\right)\right)
$$

As an example of the performance of the SCC, we examine here ACMs that link the two limits of eqs 7 and 8 . As said, we focus on noncovalent interactions because the fragments $A_{i}$ have a nondegenerate ground state, which should guarantee size consistency of the input ingredients. ${ }^{42,43}$ Moreover, in this case, the interaction energy is small, and therefore, the correction can be relevant; for covalent interactions, in fact, the correction is of the same order of magnitude as that for noncovalent ones, but the interaction energy is at least 2 orders of magnitude larger. All calculations have been performed using a development version of the TURBOMOLE package, ${ }^{51,52}$ with computational details similar to those of refs 48 and 53, in which the ISI-like functionals are evaluated on HF orbitals (see section IV.D of ref 53 for some discussion of this choice). Thus, in eq $7, W_{0}[\rho]=E_{\mathrm{x}}^{\mathrm{HF}}[\rho]$ and $W_{0}^{\prime}[\rho]$ is twice the second-order Möller-Plesset (MP2) correlation energy, $W_{0}^{\prime}[\rho]=2 E_{\mathrm{c}}^{\mathrm{MP} 2}$. The strong-coupling functionals $W_{\infty}[\rho]$ and $W_{\infty}^{\prime}[\rho]$ of eq 8 are approximated with the point charge plus continuum (PC) semilocal model, ${ }^{54}$ which is reasonably accurate. ${ }^{28,45}$ We test different interpolation formulas that have been proposed in the literature, namely, SPL, ${ }^{23}$ rev-ISI, ${ }^{28}$ and LB. ${ }^{46}$ Additionally, we also tested the Padé $[1,1]$ formula of eq 6 by using $\lambda_{p}=\infty$. The interpolation formulas and additional computational details are reported in the Supporting Information.
As a first example, in Figure 1, we show the absolute errors in the interaction energy for a set of dispersion complexes made of

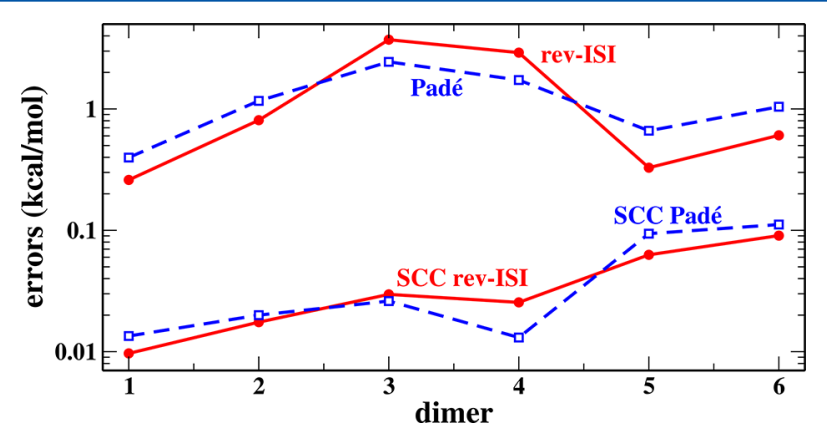

Figure 1. Absolute errors in the interaction energy ( $\mathrm{kcal} / \mathrm{mol}$, logscale) for a set of dispersion heterodimers containing noble gas atoms obtained with the rev-ISI and Padé([1/1]) functionals with and without inclusion of the SCC of eq $13(1: \mathrm{He}-\mathrm{Ne} ; 2: \mathrm{He}-\mathrm{Ar} ; 3: \mathrm{Ne}-$ $\mathrm{Ar}$; 4: $\mathrm{Ar}-\mathrm{Kr}$; 5: $\mathrm{CH}_{4}-\mathrm{Ne}$; 6: $\left.\mathrm{C}_{6} \mathrm{H}_{6}-\mathrm{Ne}\right)$.

fragments of different species obtained from the rev-ISI and the Padé interpolation formulas, computed with and without the SCC. From this figure, we can see that in both cases the error is reduced by an order of magnitude when the correction is applied, i.e, when eq 14 is used.

In Figure 2, we also report the interaction energy curve for $\mathrm{He}-\mathrm{Ne}$ obtained from the rev-ISI functional. We see that the

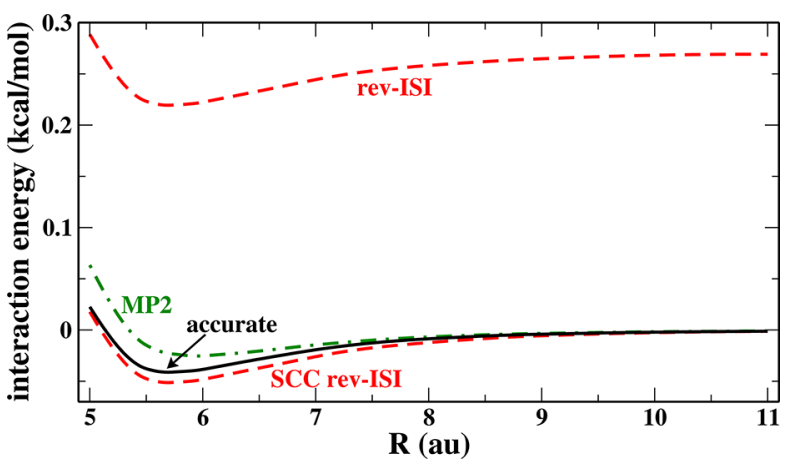

Figure 2. Interaction energy curve for the HeNe heterodimer obtained with the rev-ISI functional with and without the SCC of eq 13. The MP2 curve is shown for comparison, and the accurate curve has been taken from ref 50 .

rev-ISI curve has a very reasonable shape, but because of the size-consistency error when computed with eq 3 , it goes to a positive value with respect to the sum of the fragment energies. Instead, when the SCC is applied, the correct asymptotic value of the dissociation curve (given by eq 11) is used to compute interaction energies. Very similar figures are obtained when we consider other interpolation formulas and other systems, with the overall shift that is sometimes positive and sometimes negative.

Finally, we use the SCC to assess the accuracy of AC-based functionals for more noncovalent complexes relevant for chemistry and biology. For this purpose, we employ the wellestablished quantum-chemical data set for noncovalent interactions $566 .^{55}$

In Figure 3, we report the values of 


$$
\delta_{i}^{\mathrm{SCC}}=\left|\frac{E_{i}^{\mathrm{rev}-\mathrm{ISI}-\mathrm{SCC}}-E_{i}^{\mathrm{ref}}}{E_{i}^{\mathrm{ref}}}\right|-\left|\frac{E_{i}^{\mathrm{rev}-\mathrm{ISI}}-E_{i}^{\mathrm{ref}}}{E_{i}^{\mathrm{ref}}}\right|
$$

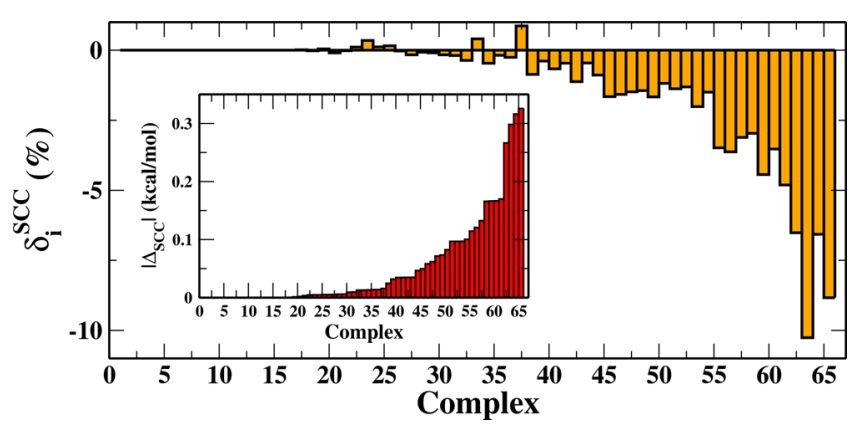

Figure 3. Difference $\delta_{i}^{\mathrm{SCC}}$ of the absolute relative errors of interaction energies calculated with and without SCC for the complexes of the S66 test set (sorted with increasing $\left|\Delta_{\text {SCC }}\right|$; see the inset).

where the index $i=1, \ldots, 66$ labels the various complexes, $E_{i}^{\text {ref }}$ is the reference interaction energy of the ith complex and $E_{i}^{\text {rev-ISI-SCC }}$ and $E_{i}^{\text {rev-ISI }}$ are the corresponding interaction energies calculated with rev-ISI with and without the SCC correction, respectively. Thus, a negative (positive) $\delta_{i}^{\mathrm{SCC}}$ means that the $S C C$ reduces (increases) the relative error of the interaction energies. Notice that in Figure 3 the $S 66$ complexes are sorted in ascending order according to the computed $\left|\Delta_{\text {SCC }}\right|$ value (see inset of Figure 3). Thus, one can see that for systems where $\left|\Delta_{\text {SCC }}\right|$ is non-negligible (i.e., $i \gtrsim 30$ ) the inclusion of SCC brings an improvement to the results $\left(\delta_{i}^{\mathrm{SCC}}<0\right)$ and that the improvement can be as large as $10 \%$. On the other hand, there are some systems (i.e., $i \lesssim 30$ ) with a negligible SCC. This is not surprising as the S66 data set contains 17 homodimers for which the AC-based functionals are already size-consistent. Moreover, there is another case in which the size-consistency error becomes negligible: when the ratio $q_{i}=W_{i}(\mathrm{~A}) / W_{i}(\mathrm{~B})$ between the $i$ th input ingredient of fragment $A$ and of fragment $\mathrm{B}$ is roughly the same for all $i, q_{i} \approx q$, a case that becomes mathematically equivalent to eq 12 . In summary, Figure 3 shows that the larger the $\Delta_{\mathrm{SCC}}$ value, the larger the reduction of the errors. This indicates that the inclusion of $\Delta_{\mathrm{SCC}}$ is significant and works correctly for most noncovalent complexes having different constituent units.

More generally, for all of the SCC-ISI-like functionals that we examined, the performance for noncovalent interactions is quite good, being comparable or better than state-of-the-art computational approaches (see Table 1). Especially for dispersion and mixed complexes, all of the ACMs perform 7 or 8 times better than the B2PLYP double hybrid and twice better than MP2 (note that both of these methods have the same computational cost as the SCC-ISI-like functionals). This is quite relevant, considering that ISI-like functionals have not been explicitly constructed to model interaction energies and do not employ any empirical parameter (in contrast, e.g., to the approaches in the last three lines of Table 1). Notice also that for AC-based functionals not only is the mean absolute error low but also the variance (last column of Table 1). Therefore, these functionals can describe different types of interactions with similar accuracy.

We have shown that XC functionals built by approximating the $\mathrm{AC}$ integrand with functions in which the input ingredients
Table 1. Mean Absolute Error (And Variance, Last Column), in $\mathrm{kcal} / \mathrm{mol}$, for the S66 Data Set and Some of Its Subsets for Different AC-Based Functionals Including SCC and Evaluated on HF Density and Orbitals ${ }^{a}$

\begin{tabular}{lccccc}
\multicolumn{1}{c}{ method } & H-bonds & dispersion & mixed & total & variance \\
rev-ISI & 0.35 & 0.44 & 0.20 & 0.33 & 0.08 \\
ISI & 0.37 & 0.42 & 0.19 & 0.33 & 0.09 \\
SPL & 0.42 & 0.42 & 0.19 & 0.35 & 0.11 \\
LB & 0.36 & 0.41 & 0.19 & 0.31 & 0.14 \\
MP2 & 0.11 & 0.81 & 0.45 & 0.45 & 0.29 \\
SCS-MI-MP2 & 0.19 & 0.45 & 0.20 & 0.19 & 0.10 \\
SCS-CCSD & 0.30 & 0.08 & 0.08 & 0.27 & 0.05 \\
B2PLYP & 0.72 & 2.79 & 1.63 & 1.71 & 1.26
\end{tabular}

${ }^{a}$ For all results, see Table S2 in the Supporting Information. The last four lines report, for comparison, results from literature. ${ }^{55,56}$

enter in a nonlinear way can be made size-consistent at no extra computational cost. The starting idea is that size consistency is restored once we consider fragments that are infinitely far apart, whose energy, by virtue of eq 11 , we compute from the sum of quantities of individual fragments. We focused here on only the case of noncovalent interactions, but the method is generally applicable also to covalent systems. We also remark that, even though in this work we only consider a few ACMs functionals, the SCC based on eq 11 has a more general applicability to any functional built using the AC framework as well as to any functional depending nonlinearly on size-consistent global quantities.

We have shown that our SCC provides in many cases an important correction to the interaction energy and leads to considerable improvement of the accuracy of various ACMs. Thus, it is a simple and efficient way to correct one of the main drawbacks of actual ACMs, which can now be reliably used for different applications. This opens the quest for the development of improved ACMs. A promising route in this direction is the construction of approximations by interpolating energy densities along the $\mathrm{AC}$, which requires nonlocal functionals for the strong-interaction limit ${ }^{35,57}$ and/or for the $\lambda=1$ case. $^{58}$

\section{ASSOCIATED CONTENT}

\section{Supporting Information}

The Supporting Information is available free of charge on the ACS Publications website at DOI: 10.1021/acs.jpclett.8b01054.

Additional details of the computations utilized in this work, additional basis functions used for each element, and results for the S66 test set (PDF)

\section{AUTHOR INFORMATION}

\section{Corresponding Author}

*E-mail: s.vuckovic@vu.nl.

\section{ORCID}

Stefan Vuckovic: 0000-0002-0768-9176

Paola Gori-Giorgi: 0000-0002-5952-1172

Fabio Della Sala: 0000-0003-0940-8830

Eduardo Fabiano: 0000-0002-3990-669X

\section{Notes}

The authors declare no competing financial interest. 


\section{ACKNOWLEDGMENTS}

This work was supported by The Netherlands Organization for Scientific Research (NWO) through an ECHO grant (717.013.004) and the European Research Council under H2020/ERC Consolidator Grant corr-DFT (Grant No. 648932).

\section{REFERENCES}

(1) Kohn, W.; Sham, L. J. Self-Consistent Equations Including Exchange and Correlation Effects. Phys. Rev. 1965, 140, A1133.

(2) Mardirossian, N.; Head-Gordon, M. Thirty years of density functional theory in computational chemistry: an overview and extensive assessment of 200 density functionals. Mol. Phys. 2017, 115, 2315-2372.

(3) Della Sala, F.; Fabiano, E.; Constantin, L. A. Kinetic-energydensity dependent semilocal exchange-correlation functionals. Int. J. Quantum Chem. 2016, 116, 1641-1694.

(4) Cohen, A. J.; Mori-Sánchez, P.; Yang, W. Challenges for density functional theory. Chem. Rev. 2012, 112, 289.

(5) Burke, K. Perspective on density functional theory. J. Chem. Phys. 2012, 136, 150901.

(6) Becke, A. D. Perspective: Fifty years of density-functional theory in chemical physics. J. Chem. Phys. 2014, 140, 18A301.

(7) Langreth, D. C.; Perdew, J. P. Solid State Commun. 1975, 17, 1425 .

(8) Gunnarsson, O.; Lundqvist, B. I. Exchange and correlation in atoms, molecules, and solids by the spin-density-functional formalism. Phys. Rev. B 1976, 13, 4274.

(9) Colonna, F.; Savin, A. Correlation energies for some two- and four-electron systems along the adiabatic connection in density functional theory. J. Chem. Phys. 1999, 110, 2828.

(10) Teale, A. M.; Coriani, S.; Helgaker, T. The calculation of adiabatic-connection curves from full configuration-interaction densities: Two-electron systems. J. Chem. Phys. 2009, 130, 104111.

(11) Teale, A. M.; Coriani, S.; Helgaker, T. Accurate calculation and modeling of the adiabatic connection in density functional theory. $J$. Chem. Phys. 2010, 132, 164115.

(12) Becke, A. D. A new mixing of Hartree-Fock and local densityfunctional theories. J. Chem. Phys. 1993, 98, 1372.

(13) Becke, A. D. Density-functional thermochemistry. III. The role of exact exchange. J. Chem. Phys. 1993, 98, 5648.

(14) Perdew, J. P.; Ernzerhof, M.; Burke, K. Rationale for mixing exact exchange with density functional approximations. J. Chem. Phys. 1996, 105, 9982-9985.

(15) Grimme, S. Semiempirical hybrid density functional with perturbative second-order correlation. J. Chem. Phys. 2006, 124, 034108.

(16) Goerigk, L.; Grimme, S. Efficient and Accurate Double-HybridMeta-GGA Density Functionalsî̀z Evaluation with the Extended GMTKN30 Database for General Main Group Thermochemistry, Kinetics, and Noncovalent Interactions. J. Chem. Theory Comput. 2011, 7, 291-309.

(17) Sharkas, K.; Toulouse, J.; Savin, A. Double-hybrid densityfunctional theory made rigorous. J. Chem. Phys. 2011, 134, 064113.

(18) Cramer, C. J.; Truhlar, D. G. Density functional theory for transition metals and transition metal chemistry. Phys. Chem. Chem. Phys. 2009, 11, 10757.

(19) Fabiano, E.; Piacenza, M.; D'Agostino, S.; Della Sala, F. Towards an accurate description of the electronic properties of the biphenylthiol/gold interface: The role of exact exchange. J. Chem. Phys. 2009, 131, 234101.

(20) Corminboeuf, C. Minimizing Density Functional Failures for Non-Covalent Interactions Beyond van der Waals Complexes. Acc. Chem. Res. 2014, 47, 3217-3224.

(21) Fabiano, E.; Constantin, L. A.; Cortona, P.; Della Sala, F. Global Hybrids from the Semiclassical Atom Theory Satisfying the Local Density Linear Response. J. Chem. Theory Comput. 2015, 11, 122-131.
(22) Ernzerhof, M. Construction of the adiabatic connection. Chem. Phys. Lett. 1996, 263, 499.

(23) Seidl, M.; Perdew, J. P.; Levy, M. Strictly correlated electrons in density-functional theory. Phys. Rev. A: At., Mol., Opt. Phys. 1999, 59, 51.

(24) Seidl, M.; Perdew, J. P.; Kurth, S. Simulation of all-order density-functional perturbation theory, using the second order and the strong-correlation limit. Phys. Rev. Lett. 2000, 84, 5070.

(25) Mori-Sanchez, P.; Cohen, A. J.; Yang, W. T. J. Chem. Phys. 2006, $125,201102$.

(26) Vuckovic, S.; Irons, T. J.; Savin, A.; Teale, A. M.; Gori-Giorgi, P. Exchange-correlation functionals via local interpolation along the adiabatic connection. J. Chem. Theory Comput. 2016, 12, 2598-2610.

(27) Cohen, A. J.; Mori-Sánchez, P.; Yang, W. Assessment and formal properties of exchange-correlation functionals constructed from the adiabatic connection. J. Chem. Phys. 2007, 127, 034101.

(28) Gori-Giorgi, P.; Vignale, G.; Seidl, M. Electronic zero-point oscillations in the strong-interaction limit of density functional theory. J. Chem. Theory Comput. 2009, 5, 743.

(29) Liu, Z. F.; Burke, K. Adiabatic connection in the low-density limit. Phys. Rev. A: At., Mol., Opt. Phys. 2009, 79, 064503.

(30) Jiang, H.; Engel, E. Orbital-dependent Representation of Correlation Energy Functional. Z. Phys. Chem. 2010, 224, 455-466.

(31) Wigner, E. P. On the interaction of electrons in metals. Phys. Rev. 1934, 46, 1002.

(32) Wigner, E. P. Effects of the electron interaction on the energy levels of electrons in metals. Trans. Faraday Soc. 1938, 34, 678.

(33) Mirtschink, A.; Seidl, M.; Gori-Giorgi, P. Energy densities in the strong-interaction limit of density functional theory. J. Chem. Theory Comput. 2012, 8, 3097.

(34) Zhou, Y.; Bahmann, H.; Ernzerhof, M. Construction of exchange-correlation functionals through interpolation between the non-interacting and the strong-correlation limit. J. Chem. Phys. 2015, 143, 124103.

(35) Bahmann, H.; Zhou, Y.; Ernzerhof, M. The shell model for the exchange-correlation hole in the strong-correlation limit. J. Chem. Phys. 2016, 145, 124104

(36) Vuckovic, S.; Irons, T. J. P.; Wagner, L. O.; Teale, A. M.; GoriGiorgi, P. Interpolated energy densities, correlation indicators and lower bounds from approximations to the strong coupling limit of DFT. Phys. Chem. Chem. Phys. 2017, 19, 6169-6183.

(37) Jaramillo, J.; Scuseria, G. E.; Ernzerhof, M. Local hybrid functionals. J. Chem. Phys. 2003, 118, 1068-1073.

(38) Maier, T. M.; Haasler, M.; Arbuznikov, A. V.; Kaupp, M. New approaches for the calibration of exchange-energy densities in local hybrid functionals. Phys. Chem. Chem. Phys. 2016, 18, 21133-21144.

(39) Vuckovic, S.; Levy, M.; Gori-Giorgi, P. Augmented potential, energy densities, and virial relations in the weak-and strong-interaction limits of DFT. J. Chem. Phys. 2017, 147, 214107.

(40) Arbuznikov, A. V.; Kaupp, M. Local hybrid exchange-correlation functionals based on the dimensionless density gradient. Chem. Phys. Lett. 2007, 440, 160-168.

(41) Bahmann, H.; Kaupp, M. Efficient Self-Consistent implementation of local hybrid Functionals. J. Chem. Theory Comput. 2015, 11, $1540-1548$.

(42) Gori-Giorgi, P.; Savin, A. Degeneracy and size consistency in electronic density functional theory. J. Phys.: Conf. Ser. 2008, 117, 012017.

(43) Savin, A. Is size-consistency possible with density functional approximations? Chem. Phys. 2009, 356, 91.

(44) For systems with a degenerate ground state, the presence of a system, even very far from another one, selects which degenerate ground state we should consider. In other words, each possible $\mathrm{M}^{*}$ selects a different set of degenerate states for the various $A_{i}$. The exact functional should be able to give the same ground-state energy for all of the degenerate states of the fragments, something that no present $\mathrm{XC}$ approximation is able to do. This is interlinked with the static correlation problem in DFT. 
(45) Seidl, M.; Gori-Giorgi, P.; Savin, A. Strictly correlated electrons in density-functional theory: A general formulation with applications to spherical densities. Phys. Rev. A: At., Mol., Opt. Phys. 2007, 75, 042511.

(46) Liu, Z. F.; Burke, K. Adiabatic connection for strictly correlated electrons. J. Chem. Phys. 2009, 131, 124124.

(47) Hobza, P.; Zahradnik, R. Intermolecular interactions between medium-sized systems. Nonempirical and empirical calculations of interaction energies. Successes and failures. Chem. Rev. 1988, 88, 871897.

(48) Fabiano, E.; Gori-Giorgi, P.; Seidl, M.; Della Sala, F. InteractionStrength Interpolation Method for Main-Group Chemistry: Benchmarking, Limitations, and Perspectives. J. Chem. Theory Comput. 2016, 12, 4885-4896.

(49) Śmiga, S.; Fabiano, E. Approximate solution of coupled cluster equations: application to the coupled cluster doubles method and noncovalent interacting systems. Phys. Chem. Chem. Phys. 2017, 19, 30249-30260.

(50) Ogilvie, J.; Wang, F. Y. Potential-energy functions of diatomic molecules of the noble gases: II. Unlike nuclear species. J. Mol. Struct. 1993, 291, 313-322.

(51) TURBOMOLE, V6.3. TURBOMOLE GmbH: Karlsruhe, Germany. http://www.turbomole.com (accessed Nov 2015).

(52) Furche, F.; Ahlrichs, R.; Hättig, C.; Klopper, W.; Sierka, M.; Weigend, F. Turbomole. WIREs Comput. Mol. Sci. 2014, 4, 91.

(53) Giarrusso, S.; Gori-Giorgi, P.; Della Sala, F.; Fabiano, E. Assessment of interaction-strength interpolation formulas for gold and silver clusters. J. Chem. Phys. 2018, 148, 134106.

(54) Seidl, M.; Perdew, J. P.; Kurth, S. Density functionals for the strong-interaction limit. Phys. Rev. A: At., Mol., Opt. Phys. 2000, 62, 012502.

(55) Rezác, J.; Riley, K. E.; Hobza, P. S66: A well-balanced database of benchmark interaction energies relevant to biomolecular structures. J. Chem. Theory Comput. 2011, 7, 2427-2438.

(56) Goerigk, L.; Hansen, A.; Bauer, C.; Ehrlich, S.; Najibi, A.; Grimme, S. A look at the density functional theory zoo with the advanced GMTKN55 database for general main group thermochemistry, kinetics and noncovalent interactions. Phys. Chem. Chem. Phys. 2017, 19, 32184-32215.

(57) Wagner, L. O.; Gori-Giorgi, P. Electron avoidance: A nonlocal radius for strong correlation. Phys. Rev. A: At., Mol., Opt. Phys. 2014, 90, 052512 .

(58) Vuckovic, S.; Gori-Giorgi, P. Simple fully non-local density functionals for the electronic repulsion energy. J. Phys. Chem. Lett. 2017, 8, 2799-2805. 